\title{
Voluntary Inhibition of Physiological Mirror Activity: An EEG-EMG Study
}

\author{
(DT. Maudrich, ${ }^{1,2}$ R. Kenville, ${ }^{1,2}$ D. Maudrich, ${ }^{1}$ A. Villringer, ${ }^{1,3,4}$ P. Ragert, ${ }^{1,2}$ and V. V. Nikulin ${ }^{1,5,6}$
}

https://doi.org/10.1523/ENEURO.0326-20.2020

${ }^{1}$ Department of Neurology, Max Planck Institute for Human Cognitive and Brain Sciences, Leipzig D-04103, Germany,
${ }^{2}$ Institute for General Kinesiology and Exercise Science, Faculty of Sports Science, University of Leipzig, Leipzig D-
04109 , Germany, ${ }^{3}$ MindBrainBody Institute at Berlin School of Mind and Brain, Charité-University Medicine Berlin,
Berlin 10099, Germany, ${ }^{4}$ Clinic for Cognitive Neurology, University Hospital Leipzig, Leipzig D-04103, Germany,
${ }^{5}$ Centre for Cognition and Decision Making, Institute for Cognitive Neuroscience, National Research University Higher
School of Economics, Moscow 101000 , Russian Federation, and ${ }^{6}$ Neurophysics Group, Department of Neurology,
Charité-University Medicine Berlin, Berlin 10117, Germany

\begin{abstract}
Physiological mirror activity (pMA), observed in healthy human adults, describes the involuntary co-activation of contralateral homologous muscles during unilateral limb movements. Here we provide novel evidence, using neuromuscular measurements (electromyography; EMG), that the amplitude of pMA can be voluntarily inhibited during unilateral isometric contractions of intrinsic hand muscles after informing human participants (10 male, 10 female) about its presence and establishing a basic understanding of pMA mechanisms through a standardized protocol. Importantly, significant suppression of pMA was observed immediately after participants were asked to inhibit it, despite the absence of any online feedback during task execution and without special training. Moreover, we observed that the decrease of pMA was specifically accompanied by an increase in relative frontal $\delta$ power recorded with electroencephalography (EEG). Correlation analysis further revealed an inverse association between the individual amplitude of pMA and frontal $\delta$ power that reached significance once participants started to inhibit. Taken together, these results suggest that $\delta$ power in frontal regions might reflect executive processes exerting inhibitory control over unintentional motor output, in this case pMA. Our results provide an initial reference point for the development of therapeutic applications related to the neurorehabilitation of involuntary movements which could be realized through the suppression of pMA observed in the elderly before it would fully manifest in undesirable overt movement patterns.
\end{abstract}

Key words: $\delta$ power; EEG; EMG; inhibition; physiological mirror activity

\section{Significance Statement}

Unilateral movements evoke unintended activation of contralateral muscles, especially when such movements are performed with high effort. This motor phenomenon, termed mirror activity (MA), is observed not only in pathologic conditions of the CNS but also in healthy individuals, where it is known as physiological MA (pMA). Surprisingly, as we show in our study, participants are capable of suppressing this unintended co-activation after being informed of its presence. Suppression was accompanied by increased $\delta$ power in frontal areas. This suggests that unintentional motor output can be suppressed via executive processes, i. e., inhibitory control. We offer an initial reference point for therapeutic applications regarding neurorehabilitation of involuntary movements and a possible strategy to counteract the age-related increase in MA.

Received July 21, 2020; accepted September 24, 2020; First published October 14, 2020.

The authors declare no competing financial interests.
Author contributions: T.M., R.K., and V.V.N. designed research; T.M., R.K., D.M., and V.V.N. performed research; T.M., R.K., D.M., and V.V.N. analyzed data; T.M., R.K., A.V., P.R., and V.V.N. wrote the paper. 


\section{Introduction}

Humans rely heavily on the bilateral independence of the limbs. A critical phenomenon that can impair such independence is termed mirror activity (MA) or associated movements (Post et al., 2009). MA involves involuntary muscle activity in the contralateral homolog of the purposely activated muscle. In severe cases, known as pathologic mirror movements (Galléa et al., 2011), this involuntary co-activation of the contralateral limb leads to overt movement that can impair even simple unilateral tasks like reaching and finger tapping. However, even in neurologically healthy adults, physiological MA (pMA) has been observed in homologous muscles (Cincotta and Ziemann, 2008). This phenomenon is hypothesized to occur because of a spillover of the initially lateralized motor command from the contralateral (controlling the active limb) toward the ipsilateral hemisphere (controlling the inactive limb), a mechanism termed motor overflow (Yensen, 1965; Perez and Cohen, 2008). Crossed facilitation within the human motor cortex, i.e., bilateral variation in the excitability of corticospinal projections, is generally observed during intentionally unilateral actions (Carson, 2005). The resulting pMA depends on the functional requirements of unilateral motor tasks (Cincotta and Ziemann, 2008). This is especially true for the level of neural drive toward the muscles engaged in the voluntary movement (Todor and Lazarus, 1986), i.e., the strength and duration of contractions (van Duinen et al., 2008; Sehm et al., 2010). Furthermore, it has been proposed that the occurrence and extent of pMA follows a U-shaped distribution across the life-span (Koerte et al., 2010). Typically, stronger pMA is observed in young children and rapidly decreases as the child enters adolescence (Mayston et al., 1999). In the third decade, pMA gradually reappears (Koerte et al., 2010). This course of pMA occurrence may reflect the processes of increasing inhibition during adolescence, with the later reappearance suggesting an age-related loss of central inhibition (Carr, 2010). Thus, it has been speculated that increased levels of pMA might be one of many altered patterns of muscle activation contributing to the decline in motor performance and manual dexterity that accompanies healthy aging (Shinohara et al., 2003).

Volitional control and suppression of involuntary movements have been demonstrated previously in patients with tremors, dyskinetic disorders, and Tourette syndrome (Koller and Biary, 1989). This suggests that

This work was supported by the Max Planck Society. V.V.N. was supported in part by the Higher School of Economics University Basic Research Program and the Russian Academic Excellence Project '5-100'.

Acknowledgements: We thank Ramona Menger, Bettina Johst, and Hartmut Domröse for organizational and technical support and Joshua Grant for proofreading.

Correspondence should be addressed to T. Maudrich at maudrich@ cbs.mpg.de.

https://doi.org/10.1523/ENEURO.0326-20.2020

Copyright (C) 2020 Maudrich et al.

This is an open-access article distributed under the terms of the Creative Commons Attribution 4.0 International license, which permits unrestricted use, distribution and reproduction in any medium provided that the original work is properly attributed. focused attention enables inhibitory mechanisms capable of controlling unintentional motor output. Based on this assumption, we hypothesized that pMA would be present when participants are unaware of such a phenomenon but can be inhibited after attention has been shifted toward its presence. Indeed, one previous investigation was able to demonstrate, in young and older adults, inhibition of contralateral muscle activity during unilateral contractions with and without visual feedback during task execution (Addamo et al., 2010). The authors used measurements of force exerted by the mirror limb to quantify pMA. However, pMA does not always lead to observable involuntary movement or torque production, which renders force measurements insufficient to capture neurophysiological aspects of pMA in its entirety.

We aim here to provide evidence for voluntary pMA inhibition based on electromyographic (EMG) measurements, allowing for the quantification of even subtle muscular activity. We propose that a possible inhibition of pMA might be mediated through higher-order cognitive control (exerted by frontal cortical regions; Addamo et al., 2010), resulting in a suppression of motor-related brain areas and finally limiting involuntary motor output. To verify this, multichannel electroencephalography (EEG) was recorded to investigate spectral power changes associated with potential inhibitory processes. Based on previous studies investigating motor inhibitory processes (Kaiser et al., 2019) and executive processes involved in lateralized inhibition of symmetric contractions (Tisseyre et al., 2019), it was hypothesized that such voluntary inhibition would be associated with increases in $\delta(1-3 \mathrm{~Hz})$ and $\theta(4-8 \mathrm{~Hz})$ power. Evidence for successful inhibition of pMA and underlying neural mechanisms could provide an initial reference point for the development of therapeutic applications aimed at involuntary movement prevention. This approach could further provide a possible strategy to counteract or prevent the increase in pMA documented in the elderly.

\section{Materials and Methods}

\section{Ethical approval}

This study was supported by the local ethics committee of the University of Leipzig (ref.-nr. 423/18-ek). All participants gave written informed consent to participate in the experiment and were compensated for involvement, according to the Helsinki Declaration.

\section{Participants}

Twenty neurologically healthy adults participated in the experiment (10 males, 10 females, age: $25.6 \pm 5.4$; mean \pm SD). All participants were right-handed according to the Oldfield handedness inventory (Oldfield, 1971). Exclusion criteria consisted of neurologic pathologies, pregnancy, centrally active drug use, regular sport, or musical participation exceeding $2 \mathrm{~h} /$ week.

\section{Experimental design}

Participants were seated comfortably in a chair and operated a custom-made force sensor with their thumb and index finger of one hand. The other hand rested on a 
Table 1: Standardized verbal instruction given during the experiment

\begin{tabular}{|c|c|}
\hline Timepoint & Instruction \\
\hline After baseline (BL) block & $\begin{array}{l}\text { The goal of this study is to investigate the so-called mirror activity phenomenon. That means that every } \\
\text { time you perform a contraction with one hand, we can also measure a weak activity in the contralateral } \\
\text { resting hand. From now on, please try to suppress this involuntary activity as much as possible by } \\
\text { a self-chosen strategy. However, keep the performance of the left hand identical. }\end{array}$ \\
\hline $\begin{array}{l}\text { After each consecutive } \\
\text { block }\end{array}$ & $\begin{array}{l}\text { Good job. However, we still see some involuntary muscle activity in your resting right hand. In the } \\
\text { following block, please try to suppress this involuntary activity again as much as possible. }\end{array}$ \\
\hline
\end{tabular}

pillow. Before starting the experiment, all participants performed a maximum voluntary contraction (MVC) test with both hands in the form of a pinch-contraction of the thumb and index finger. Three 5-s maximum contractions per hand were conducted, with 30 s of rest between each. The trial with the largest peak force was selected as the individual's MVC. This was done to normalize EMG amplitudes during data processing and scale the required force level of the following experiment $(40 \% \mathrm{MVC})$ to the individual strength capabilities. Subsequently, participants had to vertically move a cursor on a computer screen (placed in $\sim 1.5-\mathrm{m}$ distance) repetitively into a target field by applying force to the sensor (set to require $40 \pm 5 \%$ $M V C$ ) with their left hand while the right hand was resting in the prone position on the pillow. The target field was visible for $5 \mathrm{~s}$ and was followed by a 5-s rest period during which muscles should be completely relaxed. Continuous force data were recorded and used to (1) control that participants reached the target field and (2) compute the duration until participants reached the target field after it appeared on the computer screen for each contraction, i.e., ramp contraction time (RCT). The first 30 contractions served as a baseline block (BL), during which participants were naive concerning the study aim, i.e., at no time during BL were they aware of, or receive feedback about, ongoing pMA. However, pMA occurrence was confirmed through continuous observation of EMG signals during task execution. Following the $\mathrm{BL}$, participants were thoroughly educated on the pMA phenomenon using standardized verbal instructions where their attention was directed toward involuntary muscle activity of their resting hand (Table 1). Afterward, participants were asked to actively inhibit the occurrence of pMA in subsequent force blocks by a self-chosen mental strategy. The goal was to minimize EMG amplitude of the non-performing hand without changing the motor performance of the left hand. Feedback about participants' inhibition of pMA was provided verbally based on online monitoring by the researcher after the completion of each block. Online feedback was not provided. That means that participants neither received online EMG feedback nor verbal instructions during contractions. Participants could see both hands at all times but were instructed to fixate their gaze on the computer screen in front of them during all contractions. In addition to the $\mathrm{BL}$, four more blocks of 30 contractions each were performed (Iblock, II-block, III-block, and IV-block), resulting in a total of 150 epochs. In between blocks, a resting period of 3 min was granted.

\section{Data acquisition}

EMG data were recorded using a BrainAmp ExG amplifier system (Brain Products $\mathrm{GmbH}$ ). Skin preparation consisted of shaving, slight abrasion of superficial keratin layers, and cleaning with alcohol. Sintered $\mathrm{Ag} / \mathrm{AgCl}$ electrodes $(4 \mathrm{~mm}$ in diameter) were used in a monopolar setup mounted on bilateral first dorsal interossei muscles of the actively contracting hand $\left(\mathrm{FDI}_{\mathrm{Vol}}\right)$ and mirror hand $\left(\mathrm{FDI}_{\mathrm{PMA}}\right)$. Reference electrodes were placed on the right and left processus styloideus radii, respectively. One ground electrode was placed on the right epicondylus lateralis humeri. This bilateral setup allowed us to capture voluntary muscle activity of the actively contracting muscles as well as involuntary occurring pMA of the homologous resting muscles in a time-locked manner. EMG data were recorded with a sampling frequency of $1000 \mathrm{~Hz}$. EEG data were acquired using a wireless 64channel EEG system (LiveAmp, Brain Products $\mathrm{GmbH}$ ) with an active electrode setup (actiCAP, Brain Products $\mathrm{GmbH}$ ). Electrodes were mounted individually on an electrode-cap to densely cover bilateral sensorimotor cortices (Fig. 1A). Ground and reference electrodes were placed on $\mathrm{AFz}$ and left processus mastoideus, respectively. Electrode impedance was kept below $5 \mathrm{k} \Omega$ throughout the experiment. Data were continuously recorded with a sampling frequency of $500 \mathrm{~Hz}$. EMG and EEG signals were segmented around the trigger at the start of every contraction performed by the participant, resulting in a total of 150 epochs each lasting $5 \mathrm{~s}$.

\section{Data processing}

All data analyses were performed in MATLAB ( $v$. R2019b, The MathWorks Inc.) using built-in functions, the BBCl toolbox (Blankertz et al., 2016), and the EEGLAB toolbox (Delorme and Makeig, 2004).

EMG signals were first decimated to $500 \mathrm{~Hz}$ after lowpass filtering [Chebyshev type I (IIR) eighth-order filter, cutoff $200 \mathrm{~Hz}$. Furthermore, EMG data were high-pass (fourth-order Butterworth, cutoff $20 \mathrm{~Hz}$ ) and band-stop filtered at $50 \mathrm{~Hz}$ (eighth-order Butterworth) to remove power-line noise. Both EMG signals ( $\mathrm{FDI}_{\mathrm{V}_{\mathrm{ol}}}$ and $\mathrm{FDI} \mathrm{PMA}_{\mathrm{pM}}$ ) were full-wave rectified, overlaid, and time locked to preserve the temporal relationship between voluntary and involuntary muscular activity. Mean EMG amplitudes of $\mathrm{FDI}_{\text {Vol }}$, as well as $\mathrm{FDI}_{\mathrm{pMA}}$, were computed by the estimation of root mean square (RMS) values (window length, $50 \mathrm{~ms}$ ). Mean EMG amplitudes of the $\mathrm{FDI}_{\text {Vol }}$ were computed over the time window defined by the externally triggered start and end of each contraction. Latency of pMA 
A

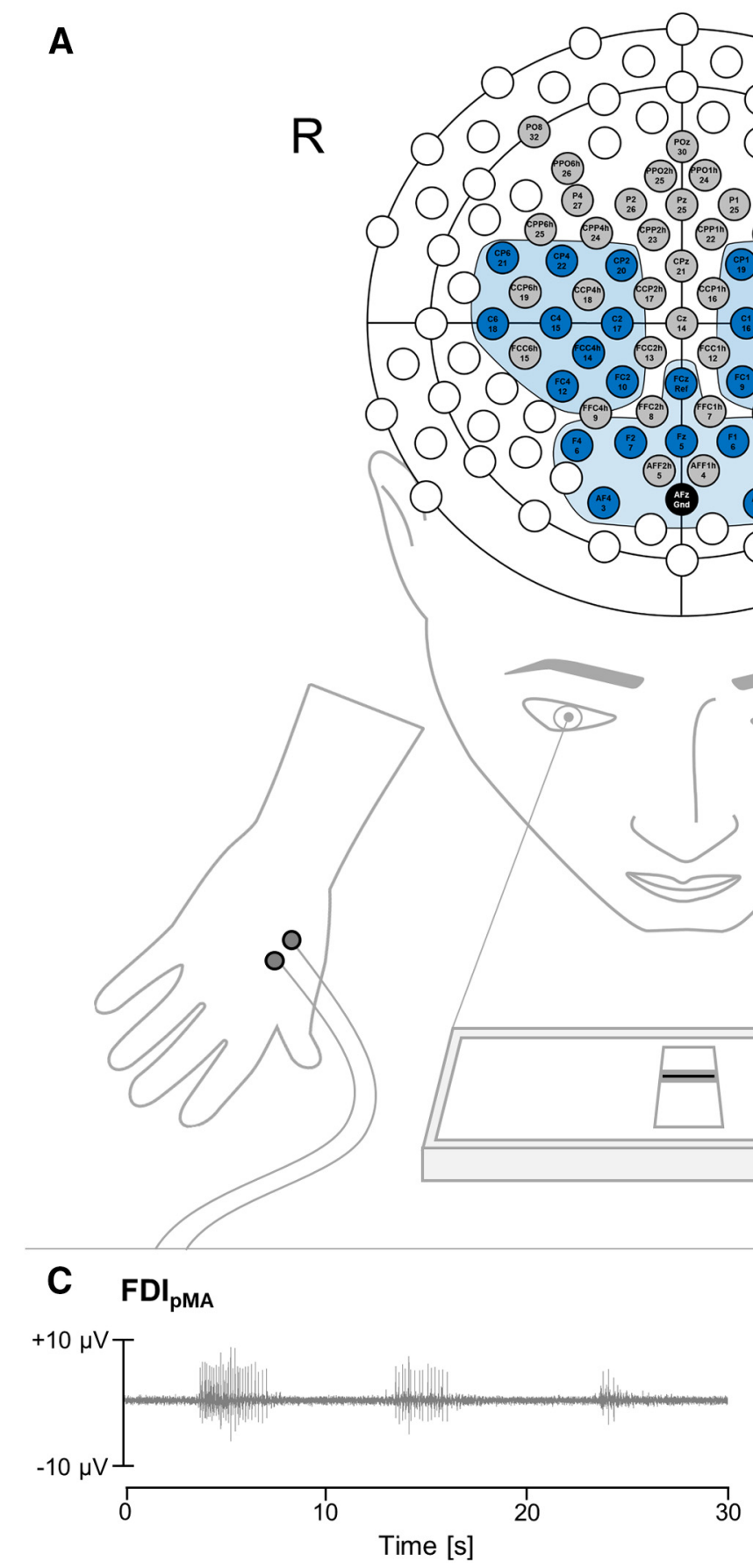

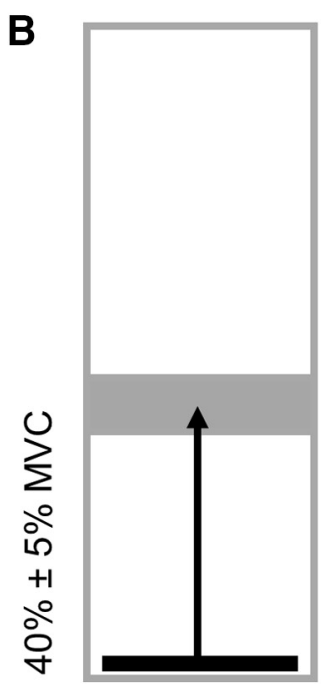

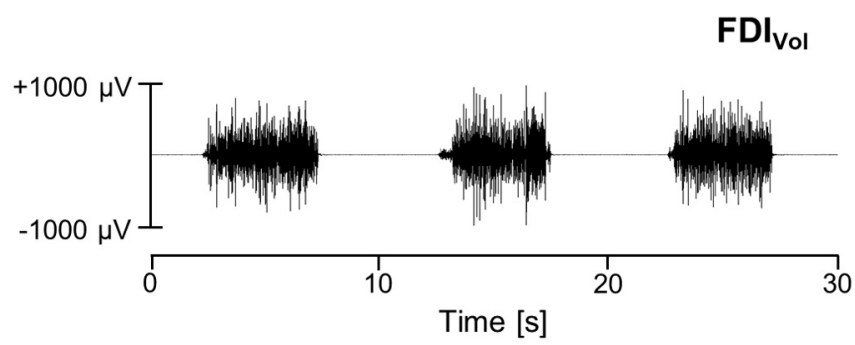

Figure 1. Experimental setup depicting the unilateral motor task combined with EEG and EMG measurements. $\boldsymbol{A}$, The motor task consisted of unilateral isometric contractions of the left hand through a custom-build force sensor while the right hand was resting in the prone position. For EEG measurements, 64 electrodes were mounted individually on a 128-channel-electrode-cap to densely cover frontal and bilateral sensorimotor cortices (blue and gray electrodes). Predefined ROls (blue electrodes): left ROI (FCC3h, FC1, FC3, C1, C3, C5, CP1, CP3, CP5), right ROI (FCC4h, FC2, FC4, C2, C4, C6, CP2, CP4, CP6), and frontal ROI (AF3, AF4, F1, F2, F3, F4, Fz, FCz). B, Participants had to vertically move a cursor on a computer screen repetitively (in total 150 times) into a target field by applying pinch force to the sensor (set to require $40 \pm 5 \%$ of MVC force). The target field was visible for $5 \mathrm{~s}$ and was followed by a 5-s rest period during which muscles should be completely relaxed. The experiment was divided into five blocks of 30 contractions each. $\boldsymbol{C}$, EMG was recorded from the bilateral FDI muscle of the voluntary contracting hand $\left(\mathrm{FDI}_{\mathrm{Vol}}\right)$ and the mirroring hand $\left(\mathrm{FDI}_{\mathrm{pMA}}\right)$. Please note the different scaling of the $\mathrm{EMG}$ traces demonstrating the subliminal nature of $\mathrm{pMA}$.

Figure Contributions: D. Maudrich designed the figure. 
was subsequently defined automatically during the delay between burst-onset of $\mathrm{FDI}_{\mathrm{Vol}}$ and the time point at which muscular activity in the contralateral (resting) $\mathrm{FDI}_{\mathrm{pMA}}$ exceeded, for a time window of at least $10 \mathrm{~ms}$, a threshold of its mean baseline activity $(1000 \mathrm{~ms}$ preFDI $_{\text {vol }}$ burst onset) +2 SD (Maudrich et al., 2019). Concerning the amplitude of pMA, latencies were taken into account so that mean EMG amplitudes of FDI were computed over the time window from the determined point of significant elevation of neuromuscular activity until the muscle offset of $\mathrm{FDI}_{\mathrm{Vol}}$. Mean EMG amplitudes were additionally estimated for pre contraction epochs (4-s rest periods between consecutive contractions) for $\mathrm{FDI}_{\mathrm{Vol}}$, as well as $\mathrm{FDI}_{\mathrm{pMA}}$. The absence of overt muscle activity in these epochs was checked and confirmed visually, i.e., the relaxation of participants between each consecutive contraction. This control condition was implemented to test for systematic fatigue-related or attention-related changes in baseline EMG amplitudes across the experiment. All EMG amplitudes were normalized with respect to individual MVC values measured at the beginning of every session for each hand separately. MVC values were estimated by the mean EMG activity over a time window of 500 ms during maximum unilateral force production for $\mathrm{FDI}_{\mathrm{Vol}}$ and $\mathrm{FDI} \mathrm{pMA}_{\mathrm{p}}$, respectively. For each participant mean amplitudes of $\mathrm{FDI}_{\mathrm{Vol}}$ and $\mathrm{FDI}_{\mathrm{pMA}}$ were averaged block wise across all 30 contractions (30 precontraction epochs) before statistical analysis. The same procedure was performed for latencies of $\mathrm{FDI}_{\mathrm{PMA}}$.

EEG data were first bandpass filtered (fourth-order Butterworth, 1-100 Hz). Subsequently, independent component analysis (infomax algorithm; Bell and Sejnowski, 1995) implemented in EEGLAB was used to remove artifact components related to eye movement, eye blinking, and muscle activity (Jung et al., 2000). On average, $5.2 \pm 2.5$ components were removed per participant. Following Laplacian spatial filtering, power spectral densities (PSDs) were calculated for 30 concatenated epochs of each block (corresponding to 30 voluntary muscle contractions), for each of the 64 EEG channels separately, employing Welch's method with a Hamming window of 500 samples and an overlap of $50 \%$. Resulting PSDs were subsequently normalized to individual total power $(1-100 \mathrm{~Hz})$. Next, relative power within distinct frequency bands of interest $(\delta: 1-3 \mathrm{~Hz}, \theta: 4-8 \mathrm{~Hz}, \alpha: 9-12 \mathrm{~Hz})$ was calculated by summation of respective frequency bins and extracted for each participant and electrode separately. Frequency bands of interest were chosen based on previous studies investigating motor inhibitory processes (Kaiser et al., 2019) and executive processes involved in lateralized inhibition of symmetrical contractions (Tisseyre et al., 2019). Subsequently, PSDs were averaged across three regions of interest (ROls; Fig. 1A): left sensorimotor $\mathrm{ROI}$ (FCC3h, FC1, FC3, C1, C3, C5, CP1, CP3, CP5), right sensorimotor ROI (FCC4h, FC2, FC4, C2, C4, C6, CP2, CP4, CP6), and frontal ROI (AF3, AF4, F1, F2, F3, F4, Fz, $\mathrm{FCz}$ ). Finally, relative power within each frequency band $(\delta, \theta, \alpha)$, block (BL-IV-block), and ROI (FRONTAL, LEFT, RIGHT) was log-transformed before statistical analyses.
The procedure was repeated for precontraction epochs (5-s rest periods between consecutive contractions) for all blocks. This control condition was implemented to test whether changes in spectral power between naive and informed blocks represent general longitudinal effects or are indeed specific to voluntary inhibitory mechanisms during the task execution.

\section{Statistical analysis}

Normality of all parameters (i.e., RCT, amplitude and latency of EMGs, log-transformed power) was assessed and confirmed through Lilliefors testing $(\alpha=0.05)$. All further statistical analyses were performed using JASP (version 0.13.1; JASP Team, 2020). Block-wise differences in RCT were investigated using a repeated-measures ANOVA with the within-subject factor BLOCK (BL, I, II, III, IV). Bonferroni adjusted $p$ values of post hoc comparisons are reported within the results. For FDI ${ }_{V o l}$ and $\mathrm{FDI}_{\mathrm{pMA}}$ (contraction and precontraction epochs), block-wise changes in amplitude were investigated using separate repeated-measures ANOVAs with the within-subject factor BLOCK (BL, I, II, III, IV). Here, the significance level was Bonferroni adjusted for four pairwise post hoc comparisons (BL vs I, BL vs II, BL vs III, BL vs IV; $\left.p_{\text {adj }}<0.0125\right)$. The same model was implemented to test for block-wise changes in latency of $\mathrm{FDI}_{\mathrm{pMA}}$. In the case of a violation of the sphericity assumption, Greenhouse-Geisser correction was implemented.

In order to compare PSD during unilateral contractions performed in a naive versus informed state, two conditions were defined for each frequency band and ROI separately: (1) power of BL-block (NAIVE) and (2) power of I-block (INHIBIT). The comparison of these two conditions reflects immediate power changes accompanying initial behavioral inhibition. Changes in log-transformed spectral power (for contraction epochs as well as precontraction epochs separately) were subsequently analyzed through two-way repeated-measures ANOVAs with the within-subject factors ROI (FRONTAL, LEFT, RIGHT) and CONDITION (NAIVE, INHIBIT) for each frequency band separately $(\delta, \theta, \alpha)$. In the case that sphericity was violated, Greenhouse-Geisser correction was implemented. Simple main effect and pairwise post hoc analyses were conducted in case of significant interaction effects.

To test our hypothesis that the amplitude of pMA observed during naive (BL) and informed blocks (I, II, III, IV) is inversely associated with the individual $\delta$ power in the frontal $\mathrm{ROI}$ for each respective block, linear regression analyses were conducted on all data per block. $\delta$ Power served as dependent variables and corresponding pMA amplitudes served as predictors. Next, we assessed normality of residuals by way of Lilliefors testing $(\alpha=0.05)$. All residuals were normally distributed. Thereafter, Pearson correlation coefficients were estimated for each block. To evaluate the significance of our results, $\delta$ power and pMA amplitude vectors were randomly permuted, resulting in a distribution of Pearson correlation coefficients from 1000 permutations (Manly, 2006). The Pearson correlation coefficients were deemed significant 
Table 2: Statistical table

\begin{tabular}{lll}
\hline Data structure & Type of test & Power \\
\hline Normal distribution & Repeated-measures ANOVA & Frequentist 95\% confidence interval \\
Normal distribution & Pearson correlation & $95 \%$ confidence interval (estimated using 1000 permutations)
\end{tabular}

when they eclipsed the 95th percentile of permuted data. Family-wise error corrected $p$ values of Pearson correlation coefficients are reported with the results. Additionally, Fisher's $r$ to $z$-transformation was implemented to test for a significant difference in the correlation coefficients of I-block and IV-block (Fisher, 1921).

The same procedure was used to estimate the association between participants' initial pMA amplitudes (BLblock) and immediate rates of inhibition [pMA amplitude BL-block-l-block (\%)].

For all statistical comparisons, $p<0.05$ was considered significant. All $p$ values Bonferroni adjusted for multiple comparisons are reported with the results.

An overview of statistical methods is provided in Table 2.

\section{Results}

\section{Behavioral parameters}

All participants consistently achieved the performance goal of the motor task by reaching the target field (corresponding to $40 \pm 5 \%$ MVC) in every 5-s contraction during the experiment (i.e., 150 contractions). Repeated-measures ANOVAs indicated a significant effect for the factor BLOCK on RCT $\left(F_{(2.1,39.9)}=11.975, p=6.660 \times 10^{-5}, \eta_{p}^{2}=\right.$ $0.387)$. Post hoc comparison showed that RCT significantly increased from $\mathrm{BL}$ to II $(\mathrm{MD}=257.098, \mathrm{SE}=48.409$, $p=1.049 \times 10^{-5}, d=1.188$ ) and stayed elevated thereafter compared with $\mathrm{BL}$ during III $(\mathrm{MD}=262.977, \mathrm{SE}=48.409$, $\left.p=6.442 \times 10^{-6}, d=1.215\right)$ and IV $(\mathrm{MD}=274.906$, $\mathrm{SE}=$ $\left.48.409, p=1.049 \times 10^{-5}, d=1.270\right)$.

\section{EMG parameters}

Repeated-measures ANOVAs indicated a significant effect for the factor BLOCK on mean amplitudes of FDI $\mathrm{Vol}$ $\left(F_{(2.517,47.832)}=10.829, p=4.092 \times 10^{-5}, \eta_{p}^{2}=0.363\right)$ and $\mathrm{FDI}_{\mathrm{pMA}}\left(F_{(1.460,27.740)}=7.140, p=0.006, \eta_{\mathrm{p}}^{2}=0.273\right)$. More specifically, post hoc analyses showed a significant increase in amplitude from $\mathrm{BL}$ to III (MD $=0.032$, $\mathrm{SE}=0.012$, $p=0.006, d=0.630)$ and $\mathrm{BL}$ to $\mathrm{IV}(\mathrm{MD}=0.070, \mathrm{SE}=0.012$, $\left.p=4.748 \times 10^{-8}, d=0.966\right)$ for $\mathrm{FDI}_{\mathrm{Vol}}(\mathrm{Fig} .2 A)$. In all blocks where participants were informed about the nature of the experiment, the amplitude of $\mathrm{FDI}_{\mathrm{pMA}}$ was significantly reduced, compared with the naive $\mathrm{BL}$ (Fig. $2 B$ ): $\mathrm{BL}$ to I $\left(\mathrm{MD}=-0.008, \mathrm{SE}=0.002, p=2.851 \times 10^{-5}, \mathrm{~d}=-\right.$ $0.652), \mathrm{BL}$ to II $(\mathrm{MD}=-0.008, \mathrm{SE}=0.002, p=4.058 \times$ $\left.10^{-5}, \mathrm{~d}=-0.645\right), \mathrm{BL}$ to III $(\mathrm{MD}=-0.07, \mathrm{SE}=0.002$, $\left.p=5.683 \times 10^{-5}, \mathrm{~d}=-0.677\right)$, and $\mathrm{BL}$ to IV (MD $=-0.006$, $\mathrm{SE}=0.002, p=0.006, d=-0.515)$. Mean EMG amplitudes of $\mathrm{FDI}_{\mathrm{Vol}}$ during precontraction epochs showed no difference between blocks, suggesting the absence of systematic changes in EMG baseline over the course of the experiment $\left(F_{(2.954,56.120)}=1.615, p=0.179, \eta_{p}^{2}=0.078\right)$.
In case of $\mathrm{FDI}_{\mathrm{pMA}}$ precontraction epochs, a significant effect of BLOCK was found $\left(F_{(2.286,43.440)}=5.327, p=0.006\right.$, $\eta_{\mathrm{p}}^{2}=0.219$ ). Corresponding to contraction epochs of $\mathrm{FDI}_{\mathrm{pMA}}$, mean EMG amplitudes were significantly reduced in all blocks where participants were informed about the nature of the experiment (except for IV-block) compared with $\mathrm{BL}: \mathrm{BL}$ to I $\left(\mathrm{MD}=-0.003, \mathrm{SE}=8.368 \times 10^{-6}, p=0.006, \mathrm{~d}=\right.$ $-0.802), \mathrm{BL}$ to II (MD $=-0.003, \mathrm{SE}=8.368 \times 10^{-6}, p=0.002$, $\mathrm{d}=-0.865)$ and $\mathrm{BL}$ to III $\left(\mathrm{MD}=-0.003, \mathrm{SE}=8.368 \times 10^{-6}\right.$, $p=0.003, d=-0.855)$. For latency of FDI $\mathrm{pMA}_{\mathrm{p}}$ no significant effect for the factor BLOCK was found $\left(F_{(4.000,76.000)}=1.456\right.$, $p=0.224, \eta_{p}^{2}=0.071$ ).

\section{$\delta$ Band}

With regards to spectral power in the $\delta$ band $(1-3 \mathrm{~Hz})$ during contraction epochs, a repeated-measures ANOVA revealed a significant interaction effect for the factors $\mathrm{ROI} \times$ CONDITION $\left(F_{(2,38)}=3.706, p=0.034, \eta_{\mathrm{p}}^{2}=0.163\right.$, Table 3). A simple main effect analysis (simple effect factor: CONDITION; moderator factor: ROI) suggests the interaction was present in the FRONTAL ROI $(p=0.003)$. Pairwise post hoc comparisons confirmed a significant increase in $\delta$ power from the NAIVE to INHIBIT condition in the FRONTAL ROI $\left(\mathrm{MD}=0.159, \mathrm{SE}=0.046, p_{\text {bonf }}=\right.$ $0.020, d=0.777$; Fig. 3). Furthermore, a significant main effect of the factor CONDITION $\left(F_{(1,19)}=5.864, p=0.026\right.$, $\left.\eta_{\mathrm{p}}^{2}=0.236\right)$ was found, with spectral power being significantly higher during INHIBIT compared with NAIVE (MD = $0.092, \mathrm{SE}=0.038, p_{\text {bonf }}=0.026, d=0.541$ ). No significant main or interaction effects were found for precontraction epochs (all $p>0.05$ ).

\section{$\theta$ Band}

In the $\theta$ band $(4-8 \mathrm{~Hz})$ no significant main or interaction effects were found, neither for contraction nor precontraction epochs (all $p>0.05$ ).

\section{$\alpha$ Band}

For the $\alpha$ band (9-12 Hz) in contraction epochs, significant main effects for $\mathrm{ROI}\left(F_{(2,38)}=31.171, p=9.720 \times\right.$ $\left.10^{-9}, \quad \eta_{\mathrm{p}}^{2}=0.621\right)$ and CONDITION $\left(F_{(1,19)}=5.946\right.$, $\left.p=0.025, \eta_{p}^{2}=0.238\right)$ were found. Pairwise post hoc analyses indicated significantly lower power in the FRONTAL ROI compared with the LEFT (MD $=-0.387$, $\left.\mathrm{SE}=0.065, p_{\text {bonf }}=2.887 \times 10^{-5}, \mathrm{~d}=-1.334\right)$ and RIGHT ROIs (MD = -0.367, SE $=0.058$, p bonf $=1.475 \times 10^{-5}, \mathrm{~d}=$ -1.406). Furthermore, a significant increase in power from the NAIVE to INHIBIT condition was revealed (MD = $\left.0.080, \mathrm{SE}=0.033, p_{\text {bonf }}=0.025, d=0.545\right)$. However, no significant interaction effect was found.

For precontraction epochs, a similar result was obtained. Significant main effects for ROI $\left(F_{(1.458,27.698)}=48.306\right.$, $\left.p=1.042 \times 10^{-8}, \quad \eta_{\mathrm{p}}^{2}=0.718\right)$ and CONDITION $\left(F_{(1,19)}=\right.$ 
A

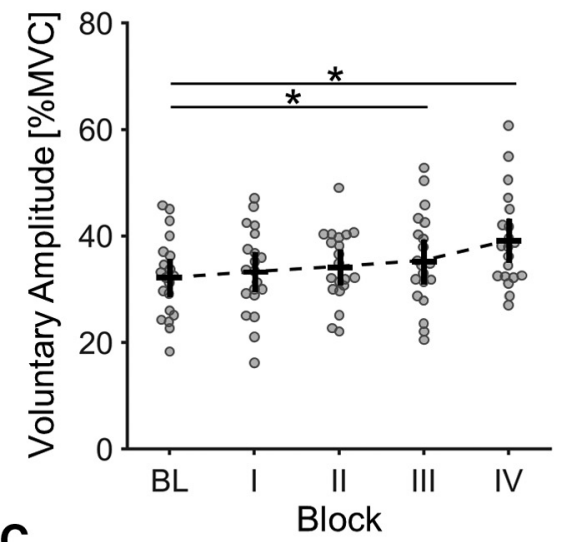

C

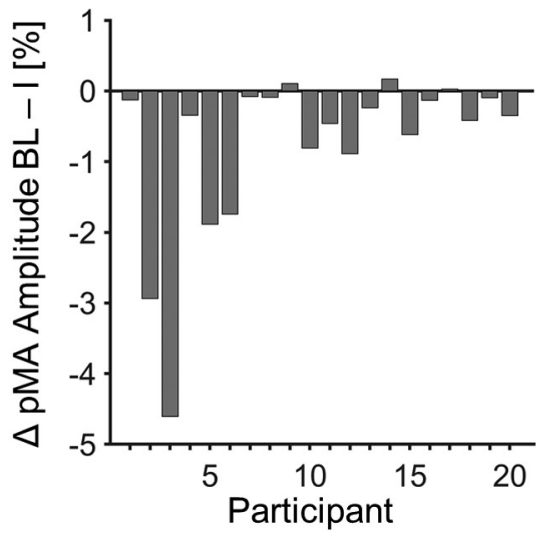

B
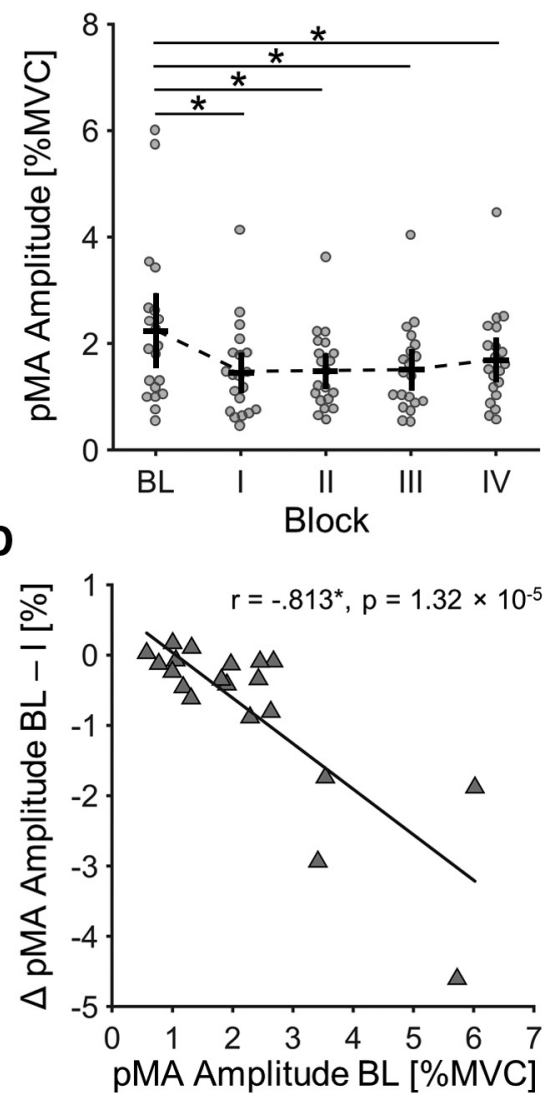

Figure 2. EMG results. A, Block-wise EMG amplitude of voluntary muscle contractions ( $\left.F D I_{\text {Vol }}\right)$. Bars around the mean indicate $95 \%$ confidence interval of the mean. Asterisks indicate significant post hoc pairwise comparisons, significance level Bonferroni adjusted to $p<0.0125$. B. Block-wise EMG amplitude of $\mathrm{pMA}\left(\mathrm{FDI}_{\mathrm{pMA}}\right)$. Bars around the mean indicate $95 \%$ confidence interval of the mean. Asterisks indicate significant post hoc pairwise comparisons, significance level Bonferroni adjusted to $p<0.0125$. $C$, Individual percentage change in amplitude of $\mathrm{FDI}_{\mathrm{PMA}}$ from BL-block to l-block for each participant. $\boldsymbol{D}$, Significant inverse relationship between initial levels of pMA (pMA amplitude in BL-block) and the percentage change in PMA amplitude from BL-block to I-block. Asterisks indicate significant correlation after permutation testing (1000 permutations). BL, baseline block; I, first inhibition block; II, second inhibition block; III, third inhibition block; IV, fourth inhibition block.

10.551, $p=0.004, \eta_{p}^{2}=0.357$ ) were found. Pairwise post hoc analyses indicated significantly lower power in the FRONTAL $\mathrm{ROI}$ compared with the LEFT (MD $=-0.447$, SE $=0.063$, $p_{\text {bonf }}=2.782 \times 10^{-6}, \mathrm{~d}=-1.589$ ) and RIGHT ROls (MD = $\left.-0.424, \mathrm{SE}=0.052, p_{\text {bonf }}=3.937 \times 10^{-7}, \mathrm{~d}=-1.818\right)$.

Table 3: Results of rmANOVAs $(n=20)$ on log-transformed PSD for each frequency band of interest: $\delta(1-3 \mathrm{~Hz})$, $\theta(4-8 \mathrm{~Hz})$, and $\alpha(9-12 \mathrm{~Hz})$

\begin{tabular}{lllrll}
\hline Freq-Band & Factor & df & \multicolumn{1}{l}{$F$} & $p$ & $\eta^{2}$ \\
\hline$\delta$ & ROI & 1.45 & 1.455 & 0.247 & 0.071 \\
& Time & 1.00 & 5.864 & $0.026^{*}$ & 0.236 \\
& ROI $\times$ Time & 2.00 & 3.706 & $0.034^{*}$ & 0.163 \\
$\theta$ & ROI & 1.33 & 0.907 & 0.379 & 0.046 \\
& Time & 1.00 & 1.627 & 0.217 & 0.079 \\
& ROI $\times$ Time & 2.00 & 0.579 & 0.566 & 0.030 \\
$\alpha$ & ROI & 2.00 & 31.171 & $9.720 \times 10^{-9} *$ & 0.621 \\
& Time & 1.00 & 5.946 & $0.025^{*}$ & 0.238 \\
& ROI $\times$ Time & 2.00 & 0.711 & 0.497 & 0.036
\end{tabular}

* significance $(p<0.05)$.
Furthermore, a significant increase in power from the NAIVE to INHIBIT condition was revealed $(\mathrm{MD}=0.091, \mathrm{SE}=0.028$, $\left.p_{\text {bonf }}=0.004, d=0.726\right)$. Again, no significant interaction effect was found.

\section{Correlation analysis}

During the naive $\mathrm{BL}$, frontal $\delta$ power and the amplitude of pMA were not significantly associated $\left(r_{(20)}=-0.340\right.$, $p=0.084)$. Once participants started to inhibit pMA, a significant negative correlation emerged during I $\left(r_{(20)}=\right.$ -0.397, $p=0.046)$, II $\left(r_{(20)}=-0.406, p=0.038\right)$, III $\left(r_{(20)}=\right.$ $-0.558, p=0.006)$ and IV $\left(r_{(20)}=-0.670, p=0.001\right.$; Fig. 4). Pearson correlation coefficient of I-block and IV-block were not significant different from each other ( $z$ score $=1.139, p=0.127$ ).

Furthermore, we found a significant negative correlation between initial levels of pMA (pMA amplitude in I-block) and the percentage change in pMA amplitude from BLblock to I-block $\left(r_{(20)}=-0.813, p=1.32 \times 10^{-5}\right)$. That means that participants with higher initial pMA amplitudes 
A

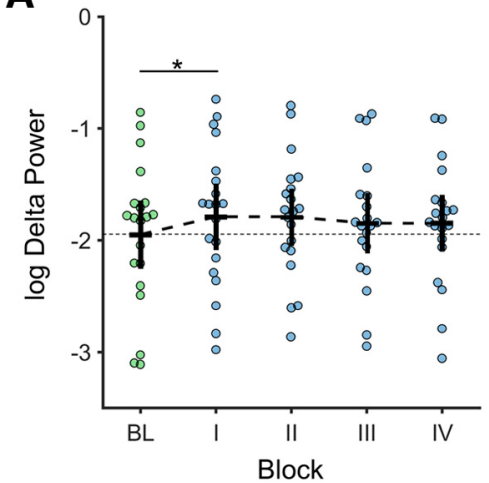

B

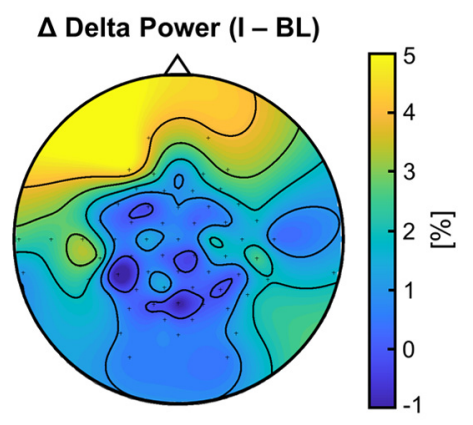

Figure 3. Spectral power change in the $\delta$ band. $\boldsymbol{A}$, Significant increase in log-transformed $\delta$ power in frontal ROI from BL-block to I-block, reflecting immediate power changes accompanying initial behavioral inhibition. Bars around the mean indicate $95 \%$ confidence interval of the mean. Asterisks indicate significant pairwise comparison. $\boldsymbol{B}$, Topographical map showing grand averaged percentage increase in $\delta$ power in frontal electrodes (contrast: I-block - BL-block). BL, baseline block; I, first inhibition block; II, second inhibition block; III, third inhibition block; IV, fourth inhibition block.

showed the highest rates of inhibition from BL-block to Iblock.

\section{Discussion}

Using a multimodal approach by combining EMG and EEG recordings, we provide novel evidence of pMA suppression in healthy adults. Suppression was accompanied by increased $\delta$ power in frontal areas. We show that the amplitude of pMA can be voluntarily inhibited during unilateral isometric contractions of intrinsic hand muscles. This was accomplished by asking participants to do so, after informing them about the presence of pMA and offering a basic understanding of pMA mechanisms through a standardized protocol. Importantly, significant inhibition of pMA was observed despite the absence of any online feedback during task execution and without special training.

A consistent and robust observation is that the amplitude of pMA is modulated by force demands of unilateral movements, i.e., increases with rising force requirements (Todor and Lazarus, 1986; Aranyi and Rosler, 2002; Shinohara et al., 2003; van Duinen et al., 2008; Sehm et al., 2010, 2016; Maudrich et al., 2017). To avoid any influence of this relationship, the force level in this study was kept constant (40 $\pm 5 \% \mathrm{MVC})$. In addition, the quality of task performance, i.e., consistently achieving the desired force level, is expected to have an influence on pMA amplitude. However, all subjects reached the target field in each trial. Together, we were, therefore, able to keep two factors that mainly influence pMA amplitude constant. Interestingly enough, a significant reduction of pMA amplitude was achieved in all informed force blocks. As we intentionally interfered with the force dependency of pMA by directing participants' attention toward the presence of pMA and asking them to inhibit any involuntary co-activation, this observation might suggest executive control, through directed attention, exerts inhibitory drive on involuntary motor output, suppressing pMA. In support of this, it has been shown in Go/No-Go reaction tasks, that primary motor cortex excitability is suppressed (Hoshiyama et al., 1996, 1997; Leocani et al., 2000), and short intracortical inhibition (SICl) is enhanced (Waldvogel et al., 2000; Sohn et al., 2002) during voluntary inhibition of movements, providing a potential neurophysiological basis for voluntary inhibition of pMA. Interestingly, we
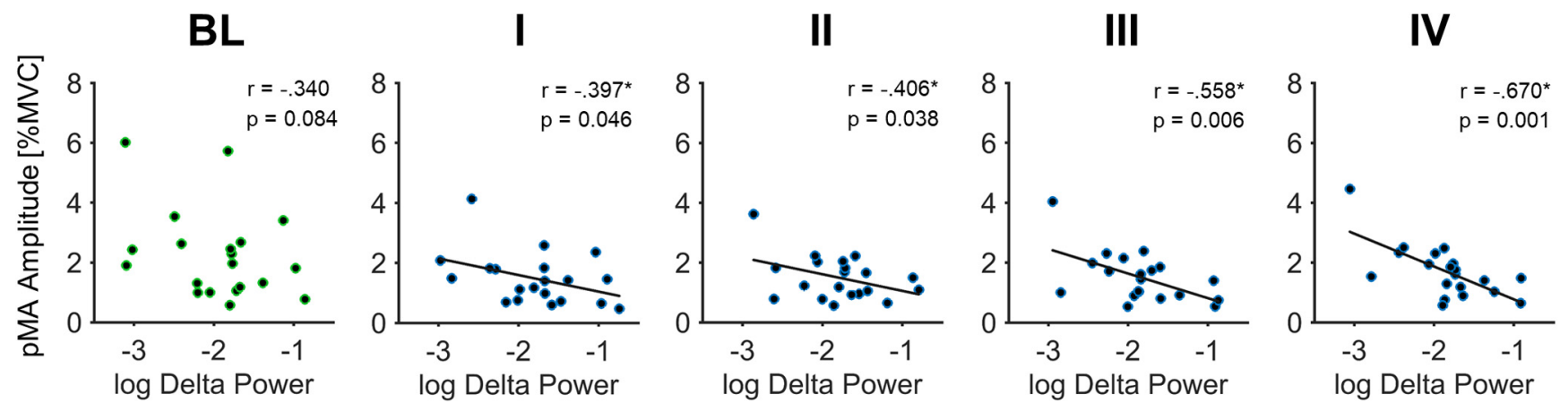

Figure 4. Inverse relationship between frontal $\delta$ power and the amplitude of pMA. Bivariate Pearson correlation depicted for each contraction block separately. The inverse association reached significance once participants were instructed to inhibit pMA (Iblock). Asterisks indicate significant correlation after permutation testing (1000 permutations). BL, baseline block; I, first inhibition block; II, second inhibition block; III, third inhibition block; IV, fourth inhibition block. 
observed different rates of inhibition between participants (Fig. 2C). In conjunction with this, we found a strong negative correlation between initial levels of pMA (pMA amplitude in I-block) and the percentage change in pMA amplitude from BL-block to I-block (Fig. 2D). This suggests that participants with higher initial pMA amplitudes showed the highest rates of inhibition. However, this correlation must be interpreted with caution. In some participants, very low initial pMA values and inhibition rates were present, suggesting on the one hand that pMA cannot demonstrate a strong decrease because of the "floor effect." On the other hand, it might be possible that the signal-to-noise ratio of the surface EMG recordings could be limiting the potential for inhibition of participants with initially low pMA amplitudes in the present experiment. Intramuscular needle EMG might be better suited to detect the most subtle involuntary discharges of motor units and could, therefore, be used in future studies to resolve the question of whether pMA can be completely eliminated.

Investigations of right-handed adults, using similar isometric contractions to our implemented task, reported no side differences in pMA between right and left-hand contractions (Addamo et al., 2009; Koerte et al., 2010; Sehm et al., 2016; Maudrich et al., 2017, 2018). Therefore, it seems reasonable to assume that the choice of the contracting hand in the current study (left), most likely did not influence the obtained result. However, future studies are needed to replicate the voluntary inhibition of pMA during dominant hand contractions in right-handed adults.

In previous investigations regarding pMA, it has been ensured that participants were naive to the study aim and generally the phenomenon of motor overflow to prevent any voluntary inhibition (Post et al., 2009; Maudrich et al., 2019). In the present experiment it seems remarkable that by shifting attention to the involuntarily contracting hand and establishing a basic understanding of pMA, most of the participants were able to significantly inhibit the amplitude of pMA in successive contraction blocks without any online feedback about their performance or special training. After the experiment, participants were asked to describe their self-chosen strategy used to inhibit pMA. While many different and highly individualized strategies have been mentioned, a main common denominator can be inferred. All participants, in one way or another, mentally focused on non-activation of the non-performing hand during each contraction of the performing hand. Thus, constantly paying attention to muscle relaxation in non-performing limbs might be a prerequisite for successful pMA inhibition besides being aware of its presence. However, no clear relation between individually employed strategies and participants inhibition rates is recognizable so that a decisive conclusion cannot be drawn from this dataset. Another strategy that was not reported but is manifested in increased RCTs during the experiment might be to perform more deliberate, less explosive contractions of the performing hand. Slower contractions of the performing hand, without changing the required target force level, might have allowed for easier suppression of pMA in the non-performing hand.
In line with the behavioral inhibition, we observed an increase in relative $\delta$ power from BL-block to the consecutive I-block in the frontal ROI (sensor space). This taskrelated increase in $\delta$ power was most likely not because of general longitudinal or non-stationarity effects during the experiment as our control comparison of non-task-related epochs (directly preceding muscle contractions) failed to reach significance. Previously, it has been hypothesized that there is a connection between $\delta$-oscillations and suppression of unwanted neural activity (Vogel et al., 1968). Furthermore, studies have shown that increases in $\delta$ power in frontal regions are associated with behavioral inhibition (Kamarajan et al., 2004; Knyazev, 2007; Putman, 2011), and it has been proposed that midfrontal $\delta$ power might serve as a selective marker for motor inhibition (Kaiser et al., 2019). Other investigations incorporating Go/No-Go tasks have observed an increase in $\delta$ activity during inhibition of movement in frontal electrodes (Harmony et al., 2009). Moreover, another study investigating the neurocognitive effects of alcoholism proposed that decreased $\delta$ and $\theta$ power in frontal regions, associated with No-Go processing, might suggest a deficient inhibitory control and information-processing mechanism in alcoholics (Kamarajan et al., 2004). In addition, we found a significant inverse association between the amplitude of pMA and frontal $\delta$ power that emerged once participants were instructed to inhibit pMA and persisted throughout all successive force blocks (Fig. 4). Overall, these results suggest that $\delta$ power in frontal regions might reflect executive processes relating to inhibitory control (Chambers et al., 2009; Munakata et al., 2011), modulating unintentional motor output, in our case pMA. Importantly, the fact that participants were able to suppress pMA immediately after they were instructed indicates that no special training was required. Thus, a reduction of involuntary motor output had rather a categorical character akin to a termination of a given behavioral output. Such regulation of behavior is a hallmark of executive functions, particularly inhibitory control, which often has a character of a veto, once undesirable consequences of the behavior become known (Kühn et al., 2009). In such a case, the over-riding of pMA is similar to not performing a Go-response in a No-Go paradigm, once the participant is made aware that she/he was committing this response but were not yet aware of such behavior. Bringing the presence of pMA to their attention thus requires them to perform a Go-response with one hand (voluntary contraction) while simultaneously preventing this response with the other hand (involuntary muscle activation).

Contrary to our hypothesis, we did not observe changes in $\theta$ power from BL-block to l-block. This seems surprising, as a previous investigation found a $\theta$ power increase associated with lateralized inhibition of symmetrical movements in the fronto-mesial area, attributed to executive processes (Tisseyre et al., 2019). This contradiction may be explained by differences between our task and those from previous studies showing $\theta$ power increases during motor inhibition. Such increases in $\theta$ power were often demonstrated during voluntary response inhibition paradigms (Cohen et al., 2011; Andreou 
et al., 2017). In this context, voluntary response inhibition is closely related to feedback on task performance, meaning that inhibitory strategies and their success rates can be assessed and optimized during task performance (Yamanaka and Yamamoto, 2010). Thus, this ability to strategically fine-tune inhibitory strategies (Kirmizi-Alsan et al., 2006; Yamanaka and Yamamoto, 2010; Tisseyre et al., 2019) is a major difference compared with the task we employed in this study. Because of the inherent characteristics of the motor task in the present study, where participants were entirely unaware regarding successful inhibition of pMA throughout the experiment, strategic fine-tuning is unlikely to have occurred because of participants' uncertainty regarding successful inhibition. This mechanistic difference may serve as an explanation for the absence of an increase in $\theta$ power as participants could not have been certain regarding successful pMA inhibition and, therefore, stable $\theta$ dynamics, reflecting voluntary inhibitory response mechanisms did not emerge.

With regards to $\alpha$-oscillations, which among other functions generally reflect inhibitory control mechanisms in the motor domain (Klimesch et al., 2007; Sauseng et al., 2013), we observed overall global power increases from naive to informed contractions regardless of $\mathrm{ROI}$. Furthermore, this global increase in $\alpha$ power was also observed in our control analysis of non-task related epochs. An increase in $\alpha$ power over time has previously been associated with the level of sustained attention and fatigue (Boksem et al., 2005; Craig et al., 2012). Recently, multiple endogenous non-stationarity processes in $\alpha$ band activity, i.e., an increase in power, have been shown to occur over time (Benwell et al., 2019). Therefore, the global $\alpha$ power increase observed in the present study most likely reflects the longitudinal effects of fatigue, sustained attention, or endogenous non-stationarity processes. However, the absence of specific changes in $\alpha$ power further supports our interpretation that inhibition of pMA in this study is mediated by executive control, rather than by purely motor inhibition processes.

As a limitation of the present study, we are not able to disentangle the distinct effects of directed focus on pMA presence and actual inhibitory processes on the observed behavioral suppression of pMA. One previous study observed that pMA remained unchanged after young adults were informed of it but asked to ignore their motor overflow. Only when participants were requested to inhibit involuntary co-activation with and without visual feedback were they able to reduce pMA (Addamo et al., 2010). While this within-subject study protocol seems appropriate to disentangle the effects of directed focus and actual inhibitory processes on pMA suppression in general, we suggest that a between-subject protocol might be an alternative. As part of this between-subject protocol, one intervention group would be informed about pMA presence but instructed to ignore it, while a separate intervention group is additionally instructed to actively inhibit pMA. A control group would be naive regarding pMA throughout the experiment. This experimental design could be repeated over several days to investigate the stability of these mechanisms or even learning effects in
pMA inhibition over time. However, as stated before, force measurements are insufficient in capturing neurophysiological aspects of pMA in its entirety, rendering future studies incorporating EMG measurements necessary to answer these questions raised above.

In conclusion, we provide novel evidence for voluntary suppression of pMA in healthy adults. As proposed by others, the knowledge of voluntary pMA modulation via attentional processes might be helpful for therapeutic applications in the context of neurorehabilitation, i.e., for the development of cognitive-behavioral strategies for the treatment of mirror movements (MM) that are not a result of major anatomic abnormalities (e.g., uncrossed corticospinal tract; Addamo et al., 2007, 2009). One possible intervention might be to implement online feedback of involuntary muscular activity to voluntarily decrease the level of EMG and even possibly the occurrence of overt $\mathrm{MM}$, as it has been demonstrated previously in brain-injured young adults (Lazarus, 1992). However, the transfer of pMA-mechanisms to pathologic MM remains elusive and requires further research. Nevertheless, this inhibitory feedback-based approach appears additionally promising in the context of counteracting the age-related increases in pMA, which might help prevent the decline in motor performance documented in the elderly.

\section{References}

Addamo PK, Farrow M, Hoy KE, Bradshaw JL, Georgiou-Karistianis $N$ (2007) The effects of age and attention on motor overflow production-A review. Brain Res Rev 54:189-204.

Addamo PK, Farrow M, Hoy KE, Bradshaw JL, Georgiou-Karistianis $N$ (2009) The influence of task characteristics on younger and older adult motor overflow. Q J Exp Psychol 62:239-247.

Addamo PK, Farrow M, Bradshaw JL, Moss S, Georgiou-Karistianis N (2010) The effect of attending to motor overflow on its voluntary inhibition in young and older adults. Brain Cogn 74:358-364.

Andreou C, Frielinghaus $\mathrm{H}$, Rauh J, Mußmann M, Vauth S, Braun P, Leicht G, Mulert C (2017) Theta and high-beta networks for feedback processing: a simultaneous EEG-fMRI study in healthy male subjects. Transl Psychiatry 7:e1016.

Aranyi Z, Rosler KM (2002) Effort-induced mirror movements. A study of transcallosal inhibition in humans. Exp Brain Res 145:7682.

Bell AJ, Sejnowski TJ (1995) An information-maximization approach to blind separation and blind deconvolution. Neural Comput 7:1129-1159.

Benwell CSY, London RE, Tagliabue CF, Veniero D, Gross J, Keitel C, Thut $\mathrm{G}$ (2019) Frequency and power of human alpha oscillations drift systematically with time-on-task. Neuroimage 192:101-114.

Blankertz B, Acqualagna L, Dähne S, Haufe S, Schultze-Kraft M, Sturm I, Ušćumlic M, Wenzel MA, Curio G, Müller K-R (2016) The Berlin brain-computer interface: progress beyond communication and control. Front Neurosci 10:530.

Boksem MA, Meijman TF, Lorist MM (2005) Effects of mental fatigue on attention: an ERP study. Brain Res Cogn Brain Res 25:107116.

Carr LJ (2010) Some reflections on mirror movements. Dev Med Child Neurol 52:1077-1078.

Carson RG (2005) Neural pathways mediating bilateral interactions between the upper limbs. Brain Res Brain Res Rev 49:641-662.

Chambers CD, Garavan H, Bellgrove MA (2009) Insights into the neural basis of response inhibition from cognitive and clinical neuroscience. Neurosci Biobehav Rev 33:631-646. 
Cincotta M, Ziemann U (2008) Neurophysiology of unimanual motor control and mirror movements. Clin Neurophysiol 119:744-762.

Cohen MX, Wilmes K, Vijver I (2011) Cortical electrophysiological network dynamics of feedback learning. Trends Cogn Sci 15:558566.

Craig A, Tran Y, Wijesuriya N, Nguyen H (2012) Regional brain wave activity changes associated with fatigue. Psychophysiology 49:574-582.

Delorme A, Makeig S (2004) EEGLAB: an open source toolbox for analysis of single-trial EEG dynamics including independent component analysis. J Neurosci Methods 134:9-21.

Fisher RA (1921) On the "probable error" of a coefficient of correlation deduced from a small sample. Metron 1:3-32.

Galléa C, Popa T, Billot S, Méneret A, Depienne C, Roze E (2011) Congenital mirror movements: a clue to understanding bimanual motor control. J Neurol 258:1911-1919.

Harmony T, Alba A, Marroquín JL, González-Frankenberger B (2009) Time-frequency-topographic analysis of induced power and synchrony of EEG signals during a Go/No-Go task. Int $\mathrm{J}$ Psychophysiol 71:9-16.

Hoshiyama M, Koyama S, Kitamura Y, Shimojo M, Watanabe S, Kakigi R (1996) Effects of judgement process on motor evoked potentials in Go/No-go hand movement task. Neurosci Res 24:427430.

Hoshiyama M, Kakigi R, Koyama S, Takeshima Y, Watanabe S, Shimojo M (1997) Temporal changes of pyramidal tract activities after decision of movement: a study using transcranial magnetic stimulation of the motor cortex in humans. Electroencephalogr Clin Neurophysiol 105:255-261.

Jung TP, Makeig S, Humphries C, Lee TW, McKeown MJ, Iragui V, Sejnowski TJ (2000) Removing electroencephalographic artifacts by blind source separation. Psychophysiology 37:163-178.

Kaiser J, Simon NA, Sauseng P, Schütz-Bosbach S (2019) Midfrontal neural dynamics distinguish between general control and inhibition-specific processes in the stopping of motor actions. Sci Rep 9:13054.

Kamarajan C, Porjesz B, Jones KA, Choi K, Chorlian DB, Padmanabhapillai A, Rangaswamy $M$, Stimus AT, Begleiter $\mathrm{H}$ (2004) The role of brain oscillations as functional correlates of cognitive systems: a study of frontal inhibitory control in alcoholism. Int J Psychophysiol 51:155-180.

Kirmizi-Alsan E, Bayraktaroglu Z, Gurvit H, Keskin YH, Emre M, Demiralp T (2006) Comparative analysis of event-related potentials during Go/NoGo and CPT: decomposition of electrophysiological markers of response inhibition and sustained attention. Brain Res 1104:114-128.

Klimesch W, Sauseng P, HansImayr S (2007) EEG alpha oscillations: the inhibition-timing hypothesis. Brain Research Rev 53:63-88.

Knyazev GG (2007) Motivation, emotion, and their inhibitory control mirrored in brain oscillations. Neurosci Biobehav Rev 31:377-395.

Koerte I, Eftimov L, Laubender RP, Esslinger O, Schroeder AS, ErtlWagner B, Wahllaender-Danek U, Heinen F, Danek A (2010) Mirror movements in healthy humans across the lifespan: effects of development and ageing. Dev Med Child Neurol 52:1106-1112.

Koller WC, Biary NM (1989) Volitional control of involuntary movements. Mov Disord 4:153-156.

Kühn S, Haggard P, Brass M (2009) Intentional inhibition: how the "veto-area" exerts control. Hum Brain Mapp 30:2834-2843.

Lazarus JC (1992) Associated movement in hemiplegia: the effects of force exerted, limb usage and inhibitory training. Arch Phys Med Rehabil 73:1044-1049.

Leocani L, Cohen LG, Wassermann EM, Ikoma K, Hallett M (2000) Human corticospinal excitability evaluated with transcranial magnetic stimulation during different reaction time paradigms. Brain 123:1161-1173.
Manly BF (2006) Randomization, bootstrap and Monte Carlo methods in biology. Boca Raton: CRC Press.

Maudrich T, Kenville R, Lepsien J, Villringer A, Ragert P, Steele CJ (2017) Mirror electromyografic activity in the upper and lower extremity: a comparison between endurance athletes and non-athletes. Front Hum Neurosci 11:485.

Maudrich T, Kenville R, Lepsien J, Villringer A, Ragert P (2018) Structural neural correlates of physiological mirror activity during isometric contractions of non-dominant hand muscles. Sci Rep 8:9178.

Maudrich T, Kenville R, Nikulin VV, Maudrich D, Villringer A, Ragert P (2019) Inverse relationship between amplitude and latency of physiological mirror activity during repetitive isometric contractions. Neuroscience 406:300-313.

Mayston MJ, Harrison LM, Stephens JA (1999) A neurophysiological study of mirror movements in adults and children. Ann Neurol 45:583-594.

Munakata Y, Herd SA, Chatham CH, Depue BE, Banich MT, O'Reilly RC (2011) A unified framework for inhibitory control. Trends Cogn Sci 15:453-459.

Oldfield RC (1971) The assessment and analysis of handedness: the Edinburgh inventory. Neuropsychologia 9:97-113.

Perez MA, Cohen LG (2008) Mechanisms underlying functional changes in the primary motor cortex ipsilateral to an active hand. $J$ Neurosci 28:5631-5640.

Post M, Bakels R, Zijdewind I (2009) Inadvertent contralateral activity during a sustained unilateral contraction reflects the direction of target movement. J Neurosci 29:6353-6357.

Putman P (2011) Resting state EEG delta-beta coherence in relation to anxiety, behavioral inhibition, and selective attentional processing of threatening stimuli. Int J Psychophysiol 80:63-68.

Sauseng P, Gerloff C, Hummel FC (2013) Two brakes are better than one: the neural bases of inhibitory control of motor memory traces. Neuroimage 65:52-58.

Sehm B, Perez MA, Xu B, Hidler J, Cohen LG (2010) Functional neuroanatomy of mirroring during a unimanual force generation task. Cereb Cortex 20:34-45.

Sehm B, Steele CJ, Villringer A, Ragert P (2016) Mirror motor activity during right-hand contractions and its relation to white matter in the posterior midbody of the corpus callosum. Cereb Cortex 26:4347-4355.

Shinohara M, Keenan KG, Enoka RM (2003) Contralateral activity in a homologous hand muscle during voluntary contractions is greater in old adults. J Appl Physiol (1985) 94:966-974.

Sohn YH, Wiltz K, Hallett M (2002) Effect of volitional inhibition on cortical inhibitory mechanisms. J Neurophysiol 88:333-338.

Tisseyre J, Marquet-Doléac J, Barral J, Amarantini D, Tallet J (2019) Lateralized inhibition of symmetric contractions is associated with motor, attentional and executive processes. Behav Brain Res 361:65-73.

Todor JI, Lazarus JA (1986) Exertion level and the intensity of associated movements. Dev Med Child Neurol 28:205-212.

van Duinen H, Renken R, Maurits NM, Zijdewind I (2008) Relation between muscle and brain activity during isometric contractions of the first dorsal interosseus muscle. Hum Brain Mapp 29:281-299.

Vogel W, Broverman DM, Klaiber EL (1968) EEG and mental abilities. Electroencephalogr Clin Neurophysiol 24:166-175.

Waldvogel D, van Gelderen P, Muellbacher W, Ziemann U, Immisch I, Hallett M (2000) The relative metabolic demand of inhibition and excitation. Nature 406:995-998.

Yamanaka K, Yamamoto Y (2010) Single-trial EEG power and phase dynamics associated with voluntary response inhibition. J Cogn Neurosci 22:714-727.

Yensen R (1965) A factor influencing motor overflow. Percept Mot Skills 20:967-968. 